Regards sur l'économie allemande

Bulletin économique du CIRAC

82 | 2007

Varia

\title{
Le marché allemand des déchets en pleine évolution
}

Rina Bohle Zeller

\section{OpenEdition}

Journals

Édition électronique

URL : http://journals.openedition.org/rea/618

DOI : $10.4000 /$ rea.618

ISBN : 978-2-8218-0860-7

ISSN : 1965-0787

Éditeur

CIRAC

Édition imprimée

Date de publication : 1 juillet 2007

Pagination : 19-24

ISSN : 1156-8992

Référence électronique

Rina Bohle Zeller, « Le marché allemand des déchets en pleine évolution », Regards sur l'économie allemande [En ligne], 82 I juillet 2007, document 3, mis en ligne le 01 juillet 2009, consulté le 01 mai 2019. URL : http://journals.openedition.org/rea/618; DOI : 10.4000/rea.618 


\section{Le marché allemand des déchets en pleine évolution}

\author{
Rina Bohle Zeller
}

\begin{abstract}
En Allemagne, l'organisation des services de déchets, comprenant le ramassage, le recyclage ou retraitement et la mise en déchetterie, se caractérise par une répartition des compétences entre le secteur public et le secteur privé - le premier prenant en charge exclusivement les déchets domestiques et le second tout autre type de déchets. L'évolution du cadre réglementaire affectant le partage des responsabilités laisse apparaître deux évolutions distinctes: tandis que les communes cherchent actuellement à se réapproprier les services de ramassage des déchets ménagers, sous-traité en large partie au secteur privé, le secteur privé se trouve en phase de restructuration avec l'entrée d'entreprises étrangères, surtout françaises. D'autres réformes réglementaires imminentes laissent entrevoir que le marché allemand des déchets reste en mouvement.
\end{abstract}

En Allemagne, l'organisation des services de collecte et de traitement des déchets ménagers relève des communes. L'art. $28 \S 2$ al. 1 de la Loi fondamentale garantit en effet à ces collectivités la souveraineté administrative concernant les activités de "prévoyance " (Daseinsvorsorge) qui, en tant que services d'intérêt général, incluent les services de collecte et de traitement des déchets ménagers, les services de l'eau ou encore les services d'énergie et le transport public (voir $R E A$ 77/06). La répartition des compétences, caractéristique pour la République fédérale, soumet la souveraineté des communes au cadre législatif fixé par le Bund et les Länder. De son côté, le Bund, également responsable pour l'adaptation des lois allemandes à la réglementation européenne et pour le droit de la concurrence, établit la législation cadre sur la protection de l'environnement dont fait partie la gestion des déchets. De leur côté, les Länder transcrivent ensuite les lois et décrets fédéraux en législation régionale. Cette dernière est finalement appliquée lors de l'établissement des statuts et décrets communaux. Du seul fait de cette répartition des compétences, la gestion des déchets implique une grande diversité de réglementations et d'acteurs institutionnels.

Le rôle et les responsabilités des communes n'ont toutefois cessé d'évoluer du fait de la modification progressive du cadre réglementaire. La protection de l'environnement ayant gagné en importance, la législation allemande est basée désormais sur le concept Vermeidung vor Verwertung vor Beseitigung: « éviter avant de recycler avant d'éliminer ». Les emballages sont considérés dorénavant comme des déchets recyclables : I'ancienne Loi sur l'élimination des déchets de 1972 (Abfallbeseitigungsgesetz) avait été abrogée puis remplacée par la Loi sur l'économie cyclique et les déchets de 1996 (Kreislaufwirtschafts- und Abfallgesetz, $K r W$-/AbfG) qui exclut les communes du ramassage et du traitement des emballages recyclables (§13 KrW/AbfG). La nouvelle loi complète ainsi le Décret sur les emballages recyclables de 1991 (Verpackungsverordnung, VerpackV, actuellement en voie révision) qui oblige producteurs et distributeurs à reprendre les emballages après leur utilisation et à en assurer le recyclage. La possibilité de déléguer ces obligations à des tiers ( $\S 6,3$ du VerpackV) a ouvert la voie au développement d'un système de tri, ramassage et retraitement parallèle à celui des autres déchets ménagers, appelé Système dual (Duales System).

Le marché des déchets domestiques (comprenant les déchets semblables de l'industrie et du commerce) connaît ainsi de jure la coexistence des responsabilités
La souveraineté administrative des communes...

... est en évolution depuis les années 1990

Coexistence des responsabilités publiques et privées 
publiques et privées. La présence du secteur privé dans la gestion des déchets n'est toutefois pas récente. Intégralement responsables des déchets industriels, les entreprises privées interviennent, en tant que sous-traitants des communes, également à une large échelle dans la gestion des déchets domestiques. Selon la Fédération du secteur du retraitement (Bund der deutschen Entsorgungswirtschaft, $B D E$ ), près des deux tiers du ramassage des déchets ménagers sont ainsi assurés par des entreprises privées. Leur rôle est particulièrement important dans les régions rurales, alors que la gestion des déchets en milieu urbain est généralement effectuée par des entreprises communales (des disparités importantes s'observent néanmoins au niveau des Länder). Avec l'introduction du Système dual, le changement du cadre législatif accroît en conséquence l'importance du secteur privé dans la gestion des déchets. Cela signifie pour les communes, bien que leur souveraineté soit maintenue, que leur responsabilité s'applique maintenant à des quantités de déchets considérablement réduites, du fait que les emballages correspondent à environ la moitié des déchets ménagers en termes de volume ou à un tiers en termes de poids.

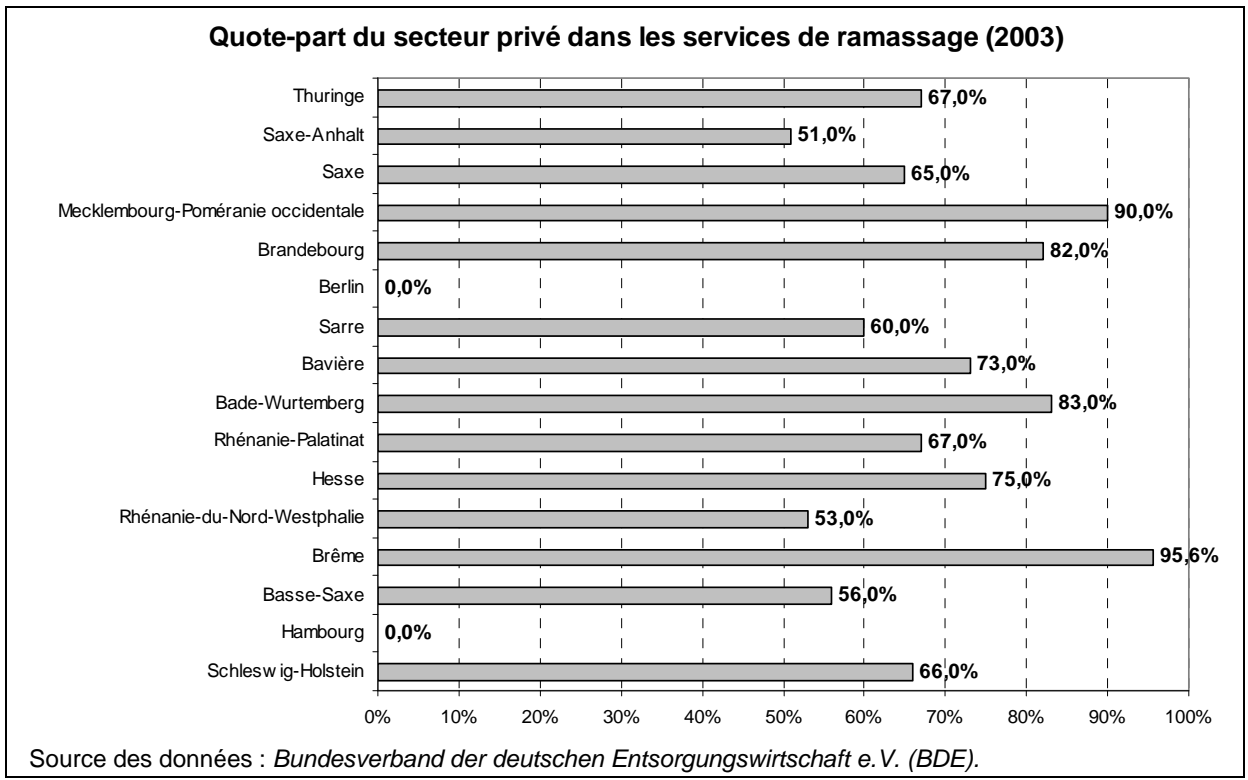

Déchets domestiques : un secteur hétérogène ...

... caractérisé par une réglementation exigeante...
Contrairement aux services de déchets domestiques en France, qui connaissent une forte concentration, outre-Rhin, l'organisation des services de ramassage, de traitement et d'élimination des déchets domestiques est très fragmentée - en fonction de la taille des structures, de leur statut juridique ou de la participation du secteur privé. Ce segment faisant partie intégrante de la Daseinsvorsorge qui incombe aux communes, et ces dernières étant souveraines dans le choix de son organisation, les services de déchets ménagers peuvent être assurés par des entreprises communales ou, en partie ou entièrement, être délégués au secteur privé ou à des sociétés d'économie mixte (§ $16 \mathrm{KrW}-/ A b f G)$. Le « principe de localité » (Örtlichkeitsprinzip) qui découle de l'art. $28 \S 2$ al. 1 de la Loi fondamentale interdit cependant aux communes d'intervenir dans la gestion des déchets hors de leur territoire ; elles ne peuvent donc pas intervenir dans l'organisation des services de déchets d'autres communes. Mais rien ne leur interdit d'élargir leur territoire en s'intégrant dans un syndicat intercommunal.

La diversité des structures opérant sur le marché des déchets ménagers s'est encore accrue avec l'évolution des exigences du cadre réglementaire. Alors qu'il existait, lors de l'adoption de la Loi sur l'élimination des déchets de 1972, environ 50000 déchetteries (hors ex-RDA), leur nombre s'est considérablement réduit depuis. Pour satisfaire aux nouvelles réglementations environnementales, les communes, tout comme le secteur privé, ont été amenées à investir dans le pré- 
traitement des déchets et dans des déchetteries modernes. Cette tendance a été renforcée par l'adoption du Décret sur le dépôt de déchets de 2001 (Abfallablagerungsverordnung) et le Décret sur les déchetteries de 2002 (Deponieverordnung). En outre, depuis juin 2005, le dépôt des déchets non traités est prohibé. Les déchetteries quant à elles doivent remplir des standards techniques élevés, visant à éviter toute contamination des nappes phréatiques et à limiter l'émission de $\mathrm{CO}_{2}$ et de méthane. De nombreuses déchetteries communales pour déchets domestiques, ne satisfaisant pas aux nouveaux standards, ont ainsi été fermées ; leur nombre est passé de 8273 en 1990 à 162 en 2006. Parallèlement, pendant que le secteur privé investissait essentiellement dans la construction d'installations de recyclage et de traitement bio-mécanique des déchets, le secteur public construisait des installations d'incinération. Du fait de l'importance de ces investissements, les communes ont souvent fait appel au secteur privé. Sur les 73 installations d'incinération recensées outre-Rhin, la moitié seulement se trouve ainsi en propriété communale, un tiers relevant du partenariat public-privé et les restantes étant gérées par le secteur privé.

Libres de choisir la forme que prend la fourniture des services de déchets domestiques, les communes choisissent le plus souvent des structures distinctes pour l'organisation du ramassage, du traitement et de l'élimination des déchets ménagers. Le ramassage, peu intensif en capital, est généralement, s’il n'est pas sous-traité au secteur privé, géré par des entreprises publiques gérées seIon les principes de gestion commerciale (Eigenbetrieb), permettant ainsi la modernisation de la gestion tout en maintenant l'exonération de l'impôt sur les sociétés et de la TVA. La construction des déchetteries et d'installations de traitement des déchets, avec un fort besoin en capital, passe régulièrement par la création d'entreprises de droit privé (le plus souvent des $\mathrm{GmbH}$ : SARL), ce qui facilite la participation du secteur privé, mais aussi le financement des installations (les emprunts d'une $\mathrm{GmbH}$ en propriété publique ne font pas partie du bilan des communes, ce qui est intéressant pour ces dernières qui connaissent souvent des situations budgétaires tendues). Lorsque les installations sont construites et gérées avec la participation du secteur privé, les communes possèdent généralement la majorité du capital (>51\%), se réservant ainsi de forts pouvoirs de contrôle et de surveillance afin d'assurer la protection de l'environnement, la qualité du service ou encore un contrôle du niveau des prix.

Du fait de la répartition des responsabilités, les entreprises publiques et privées ne s'affrontent que sur le marché des déchets ménagers, celui des déchets de l'industrie et l'agriculture étant intégralement aux mains du secteur privé. Le temps de la concurrence «pour les déchets » initiée par les déchetteries publiques avant l'entrée en vigueur des réglementations sur le dépôt des déchets et les déchetteries, est aujourd'hui révolu. Faisant face depuis l'obligation de recyclage des emballages à des volumes de déchets ménagers plus réduits (34 millions de tonnes en 1999 contre 18 seulement en 2004), les communes peinaient en effet à remplir les capacités de leurs déchetteries. Ces dernières offraient alors des réductions de prix pour des déchets du commerce et de l'industrie afin de couvrir au moins une partie des coûts fixes élevés. Dorénavant, la concurrence public-privé se limite à une compétition indirecte lorsqu'une commune décide de faire participer le secteur privé à la fourniture de ses services.

Le débat sur la libéralisation du secteur des déchets ménagers connaît, en raison de la répartition des compétences, une moindre ampleur que par exemple celui concernant les services de l'eau (voir REA 77/06). Il se polarise autour de la souveraineté des communes dans le cadre de la réforme du fédéralisme et de divers avantages en matière fiscale et dans le domaine de la concurrence d'autre part. Surtout depuis l'augmentation de la TVA (passée de $16 \%$ à $19 \%$ le $1^{\mathrm{er}}$ janvier 2007), l'exonération des communes de la TVA est considérée par le secteur privé comme une distorsion de concurrence, permettant ainsi aux entreprises publiques d'offrir leurs services à un moindre prix. La BDE projette actuellement de déposer une plainte contre cette exonération des communes
... et une multitude de structures

Secteurs publique et privé - en concurrence?

Conflit d'intérêts sur la libéralisation du secteur... 
... du fait d'une 'recommunalisation' des services de déchets

Elle devrait être limitée au ramassage de déchets ménagers auprès de la Cour Européenne de justice dans l'espoir qu'un traitement fiscal égal amènerait plus de communes à sous-traiter les services de déchets au secteur privé. Le secteur privé considère comme une autre distorsion de concurrence la possibilité qu'ont les communes de contourner l'obligation d'émettre des appels d'offre en confiant la fourniture d'un service de la Daseinsvorge directement à une entreprise publique ou un syndicat intercommunal. La Fédération d'entreprises communales (Verband kommunaler Unternehmen, VKU) pour sa part, s'appuyant sur des décisions récentes de la Cour Européenne de justice de prononcer un non-lieu dans les plaintes déposées à ce sujet, y voit une ingérence dans la souveraineté administrative des communes. En ce qui concerne l'exonération de la TVA, la VKU précise que les entreprises publiques ne peuvent déduire la TVA payée sur les achats comme le font les entreprises privées, ce qui annulerait les possibles distorsions à la concurrence.

Le conflit des intérêts privés et publics gagne du terrain depuis que la tendance à inclure le secteur privé dans le ramassage des déchets ménagers semble s'inverser : un grand nombre de communes ne prolonge pas les concessions accordées au secteur privé et réincorpore le ramassage dans des structures publiques. Selon la presse allemande, environ 100 communes, dont Hanovre et Lüneburg, ont choisi cette voie, et d'autres pourraient bientôt suivre. Le taux de participation du secteur privé, estimé par la BDE à $63 \%$ en 2003 (moyenne fédérale), pourrait alors connaître une baisse importante dans les années à venir. Le choix des communes peut s'expliquer par la profitabilité du marché du ramassage (et du tri) de déchets, présentant des marges de 8 à $12 \%$, mais aussi par le fait qu'une participation du secteur privé nécessite aujourd'hui la publication d'appels d'offre à l'échelle européenne (beaucoup de concessions, conclues généralement pour une durée de 3 à 5 ans, arrivent à échéance). Selon la Division déchets et propreté de la VKU (Verband kommunale Abfallwirtschaft und Stadtreinigung im $V K U$ ), les communes préféreraient maintenir la création de valeur et les postes de travail sous leur responsabilité et sur leur territoire. Beaucoup de communes ne prolongeraient d'ailleurs pas les concessions parce que la qualité du service des sociétés privées aurait été peu satisfaisante ou les prestations trop chères. Ces arguments sont difficiles à évaluer du fait de l'hétérogénéité des services et des structures. L'évolution des prix des services de déchets, très forte au cours des années 1990 du fait de la construction de nouvelles installations, connaît depuis 2000 le même rythme que les prix à la consommation, mais les chiffres masquent des variations souvent importantes à l'échelle régionale. Selon l'association fédérale des centrales des consommateurs (Verbraucherzentrale Bundesverband., vzbv), les tarifs annuels varient globalement entre 134 et $280 €$, mais la différence peut aller jusqu'à $500 \%$ au sein d'un Land. En Rhénanie-du-Nord-Westphalie, par exemple, les prix pour une poubelle d'une capacité de 240 litres allait en 2005 de 150 à $900 €$.

Les tendances à la 'recommunalisation' doivent cependant être considérées avec prudence. Bien qu'il existe des évolutions semblables dans d'autres secteurs de la Daseinsvorsorge (Dortmund et Bochum ont ainsi racheté Gelsenwasser, entreprise offrant des services de l'eau), la réincorporation dans les services publics devrait être limitée aux services de ramassage. La situation budgétaire des communes, quoique en amélioration depuis peu (leurs recettes ont enregistré une hausse de 1,75 milliards $€$ en 2006), ne leur permet toutefois pas de racheter ou de construire des installations de traitement ou d'incinération de déchets. Dans ces domaines, la tendance à la participation du secteur privé devrait se maintenir. Et du fait que les services de ramassage dans les régions urbaines sont traditionnellement aux mains des communes, cette recommunalisation concerne davantage les régions rurales. Mais cette option ne peut être viable pour toutes les communes rurales, l'Örtlichkeitsprinzip étant en conflit avec les économies d'échelle (les activités des entreprises privées ne sont pas limitées à un territoire spécifique). Néanmoins, les responsabilités des communes pourront connaître d'importants changements avec la redéfinition en 
cours du droit communal à la suite de la réforme du fédéralisme politique. En matière de droit environnemental, cette dernière confère aux Länder en effet plus de compétences législatives, ce qui amplifiera davantage les disparités du droit communal à l'échelle de l'Allemagne. Alors que la Bavière et le BadeWurtemberg semblent vouloir maintenir la souveraineté administrative des communes, la réforme du droit communal projetée par la Rhénanie du-NordWestphalie pourrait rendre plus difficile toute activité commerciale des communes - au bénéfice du secteur privé.

\section{Le flux de gestion des déchets}

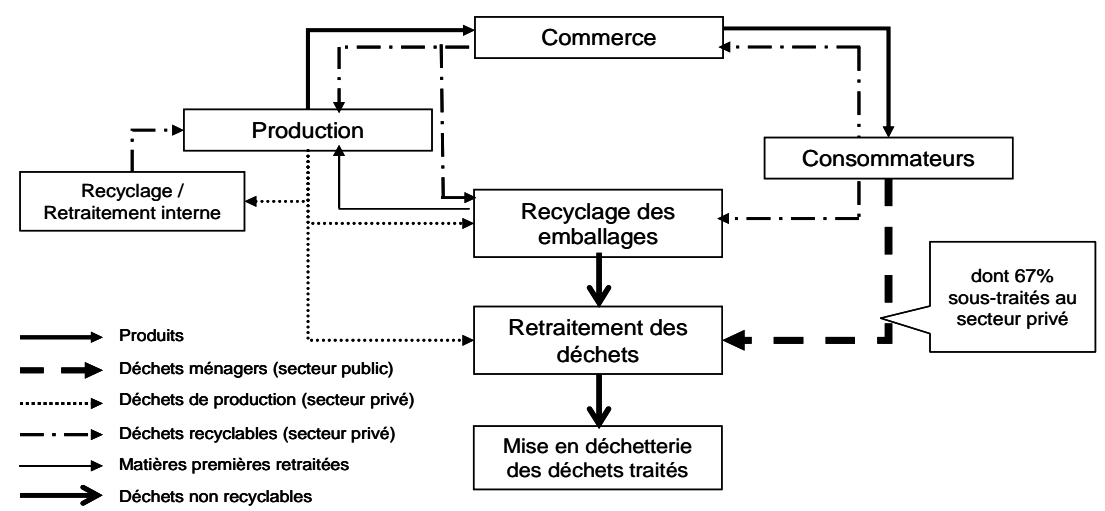

Source : Bundesumweltministerium (adapté par l'auteur)

Le marché privé des déchets (hors déchets domestiques), entièrement libéralisé, reste, malgré une tendance à la concentration, toujours caractérisé par une multitude d'entreprises se distinguant par la taille et les champs d'activités. Les services de collecte (en sous-traitance des communes), de tri, de recyclage et d'élimination se trouvent encore essentiellement aux mains de PME, dont seulement une douzaine opère à l'échelle suprarégionale (et seulement $1 \%$ des quelque 3000 entreprises recensées réalise un CA supérieur à 100 millions $€$ ). Contrairement à la France ou à l'Espagne, où les trois premières entreprises du secteur comptabilisent une part de marché de 50 à $60 \%$, cette part est de $17 \%$ seulement pour les trois premières entreprises allemandes: Remondis, Sulo/ Véolia et Alba/Interseroh. Les fusions concernent davantage les grands du secteur : Remondis avait repris $70 \%$ de RWE Umwelt en 2004 ; Sulo après avoir racheté son concurrent Cleanaway fin 2006, sera prochainement reprise par Véolia ; et l'entreprise berlinoise Alba s'est hissée à la troisième place en acquérant $46 \%$ de Interseroh. Mais cette tendance à la concentration commence à s'étendre aux grandes PME qui cherchent à se maintenir sur ce marché plus concentré. A l'instar de Tönsmeier, Sita-Wagner ou Schönmackers, elles sont aujourd'hui considérées comme les prochains acheteurs potentiels - à moins qu'elles ne deviennent à leur tour des proies pour les grands du secteur.

La tendance à la concentration est essentiellement motivée par la hausse des prix des matières premières issues du retraitement des déchets (en raison de la hausse de prix de matières premières originales), mais aussi par l'imminence de la réforme du décret VerpackV, qui engendrerait une croissance du marché du recyclage d'environ 500 millions $€$ par an. Attirées par ces perspectives, des entreprises comme Remondis et BellandVision projettent de s'établir comme opérateurs de systèmes duaux alternatifs. Des marges relativement faibles et la nécessité de pouvoir organiser les différentes structures de recyclage limiteront cependant le nombre des candidats possibles. Remondis pourrait devenir l'opérateur d'un système dual à large échelle, du fait que le groupe dispose déjà des moyens de ramassage et de retraitement. La nécessité de pouvoir proposer à des grandes chaînes de distribution (comme des supermarchés discount) des solutions à échelle fédérale limitera encore le nombre de candidats possibles.
Restructuration du marché privé des déchets?

La réforme du VerpackV, une menace pour les PME ? 


\section{Les Systèmes duaux et la réforme du VerpackV}

Les obligations du décret sur les emballages recyclables - VerpackV - de 1991 ont donné naissance au développement de systèmes de reprise, de tri et de recyclage des emballages qui opèrent parallèlement aux services de déchets ménagers (appelés de ce fait systèmes duaux).

La mise en place d'un premier système dual avait été confiée à Duales System Deutschland $\mathrm{GmbH}$ (DSD), une entreprise créée ad hoc en 1990 (voir REA 62/03) et détenue depuis 2004 par le fonds Kohlberg Kravis Roberts (KKR). Les producteurs d'emballages et le commerce versent des frais de licence au DSD, ce qui leur donne le droit d'apporter à leurs emballages le logo du DSD : « le Point Vert » (Der Grüne Punkt). Ces frais de licence permettent de financer la reprise, le tri et le recyclage des déchets portant le Point Vert. Le DSD, en tant qu'entité à but non lucratif, ne prend pas activement part dans le processus de recyclage, mais confère ces tâches à environ 550 entreprises sous-traitantes. Tenant initialement le monopole sur le marché du recyclage des emballages, le DSD a dû ouvrir ce dernier en 2004 à d'autres systèmes duaux (dont Interseroh et Landbell). Par la suite, du fait de contrôles insuffisants, le recyclage des emballages a connu des abus de la part des producteurs et distributeurs d'emballages (en cas de résiliation d'un contrat de licence avec le DSD, il est en effet difficile de prouver que l'entreprise a contracté une licence avec un de ses concurrents - il ne peut ainsi pas être garanti que l'intégralité des producteurs paient pour le recyclage de leurs emballages).

De ce fait, les systèmes duaux accueillent la réforme de la VerpackV de manière positive. Le nouveau décret, dont l'entrée en vigueur est prévue pour l'automne 2007, doit en effet garantir que seules les entreprises pouvant prouver qu'elles se sont acquittées des frais de licence à un des systèmes duaux pourraient vendre leurs produits dans le commerce.

La Fédération des entreprises qui interviennent dans la récupération des matières premières et dans le recyclage des emballages (Bundesverband Sekundärrohstoffe und Entsorgung, BVSE) craint que le marché du recyclage ne connaisse une situation d'oligopole au détriment des PME. Celles-ci, qui opèrent souvent comme sous-traitants dans un des systèmes duaux existants, pourraient être poussées vers la sortie du marché ou réduites à servir comme support logistique aux grandes entreprises du secteur détenant des installations de recyclage.

LE SECTEUR ALLEMAND DES DECHETS EST LOIN D'ETRE STABILISE. L'évolution attendue du cadre réglementaire (révision du VerpackV, la redéfinition du droit communal à la suite de la réforme du fédéralisme, etc.) devrait influer sur le rôle des communes et leur politique de 'recommunalisation', mais aussi leur ouvrir de nouvelles perspectives de croissance. L'objectif de la politique environnementale allemande, à savoir éviter la mise en déchetterie jusqu'en 2020 au bénéfice d'un recyclage et retraitement complet de tous les déchets, donnera lieu à la création de nouveaux marchés innovants. Et même si la tendance actuelle à la concentration devrait bientôt ralentir, le nombre de PME à reprendre s'étant considérablement réduit, l'intérêt des entreprises étrangères et notamment françaises restera soutenue - justement en raison des perspectives de croissance. Selon la presse allemande, Veolia (devenue après l'achat de Sulo la deuxième entreprise du marché allemand des déchets) serait aujourd'hui intéressé par la reprise de la DSD. En même temps, les entreprises allemandes (comme Remondis) s'orientent vers l'étranger, considérant notamment les nouveaux Etats membres comme des marchés prometteurs. L'organisation du marché allemand des services des déchets ne fait que commencer, préfigurant, dans un futur proche, un marché communautaire probablement dominé par quelques grands acteurs européens.

\section{Indications bibliographiques :}

BUNDESMINISTERIUM Für UMWELt, NATURSCHUTZ UND REAKTORSICHERHEIT, Abfallwirtschaft in Deutschland - Motor für Jobs und Innovationen, Berlin, juillet 2006

TOMERIUS S., "Örtliche und überörtliche wirtschaftliche Betätigung kommunaler Unternehmen ", networks working paper $n^{\circ} 6$, Berlin, septembre 2004

BUNDESMINISTERIUM FÜR UMWELT, NATURSCHUTZ UND REAKTORSICHERHEIT, Siedlungsabfallentsorgung in Deutschland, Berlin, septembre 2006

BOURGEOIS, I., "La guerre de la consigne sur les cannettes ", Regards sur l'économie allemande, $n^{\circ} 62$, juillet 2003

RAHMEYER F., Abfallwirtschaft zwischen Entsorgungsnotstand und Überkapazitäten, Coll. Volkswirtschaftliche Diskussionsreihe n²66, Universität Augsburg, novembre 2004. 